\title{
Multisystem Inflammatory Syndrome with Features of Atypical Kawasaki Disease during COVID-19 Pandemic
}

\author{
Abdul Rauf $^{1}$ (D) $\cdot$ Ajay Vijayan ${ }^{1} \cdot$ Shaji Thomas John ${ }^{1} \cdot$ Raghuram Krishnan $^{2} \cdot$ Abdul Latheef $^{3}$ \\ Received: 8 May 2020 / Accepted: 15 May 2020 / Published online: 28 May 2020 \\ (C) Dr. K C Chaudhuri Foundation 2020
}

\begin{abstract}
There is a global concern of increasing number of children presenting with inflammatory syndrome with clinical features simulating Kawasaki disease, during ongoing COVID-19 pandemic. The authors report a very similar case of 5-y-old boy from a COVID-19 hotspot area who presented in late April 2020 with acute febrile illness with abdominal pain and loose stools followed by shock. On examination, child had bulbar conjunctivitis and extremity edema. Initial investigations showed high inflammatory parameters, elevated serum creatinine and liver enzymes. Echocardiography showed moderate LV dysfunction and normal coronaries. Cardiac enzymes were also elevated, suggesting myocarditis. He was treated with inotropic support, respiratory support with high flow nasal cannula, IV immunoglobulins, aspirin, steroids and diuretics. RT PCR for SARS-CoV-2 was negative twice. His clinical condition improved rapidly, was afebrile from day 2, inflammatory parameters decreased, left ventricular function improved and was discharged after $6 \mathrm{~d}$ of hospital stay.
\end{abstract}

Keywords Kawasaki disease · Inflammatory syndrome · COVID-19 · Multi-organ dysfunction · Children

\section{Introduction}

Kawasaki disease (KD) is the most common systemic vasculitis in children, predominantly affecting the medium sized and small vessels [1]. The exact etiology of KD being not clear, it is believed that some infectious agent can trigger clinically apparent disease in individuals with certain genetic predisposition [2].

COVID-19 infection in children is less severe and has lesser mortality, compared to adults. However, National Health System (NHS) of United Kingdom and Pediatric Intensive Care Society (PICS) issued an alert recently regarding occurrence of around 20 cases of so called Pediatric multisystem inflammatory syndrome temporally associated with COVID19 [3]. This syndrome shared overlapping features with other

Abdul Rauf

abdrauf06@gmail.com

1 Department of Pediatrics, Baby Memorial Hospital, Calicut, Kerala 673004, India

2 Department of Cardiology, Baby Memorial Hospital, Calicut, Kerala, India

3 Department of Pediatrics, Santi Hospital, Omassery, Calicut, Kerala, India pediatric inflammatory conditions like KD and toxic shock syndromes. The authors report a very similar case of 5-y-old boy from a COVID infection hotspot area in Kerala state of India who presented in April 2020 with multi- organ dysfunction.

\section{Case Report}

A previously well 5-y-old boy presented with acute febrile illness without any obvious foci. On day 3 of illness, a urine routine examination showed pyuria and he was started on oral antibiotics. He continued to have high grade fever spikes and developed severe crampy abdominal pain with loose stools on day 5. USG abdomen done in a peripheral hospital for evaluation of acute abdomen was normal. As the symptoms persisted and he became lethargic, he was referred to authors' centre. On examination, he had non-purulent bulbar conjunctivitis and nonpitting edema of hands and feet. Vitals examination showed tachycardia (HR-130) and hypotension with wide pulse pressure (BP- 66/32 mmHg), suggesting vasoplegia. Complete blood count indicated neutrophilic leucocytosis [TLC- $11000 / \mu \mathrm{L}(\mathrm{N}-79 \%, \mathrm{~L}-16 \%)$ ] with normal platelet count $(3 \mathrm{lakh} / \mu \mathrm{L})$. Inflammatory parameters were high (CRP- $120 \mathrm{mg} / \mathrm{L}$, ESR $70 \mathrm{~mm} / \mathrm{h}$, Ferritin $600 \mathrm{ng} / \mathrm{ml}$ ) 
and serum creatinine $(1.3 \mathrm{mg} / \mathrm{dl})$ and liver enzymes were elevated (AST- $85 \mathrm{U} / \mathrm{L}$, ALT- $60 \mathrm{U} / \mathrm{L}$ ). Serum albumin was low $(2.1 \mathrm{~g} / \mathrm{dl})$ and hyponatremia $(124 \mathrm{mEq} / \mathrm{L})$ was also present. 2D Echocardiogram revealed global left ventricular hypokinesia with moderate systolic dysfunction (Ejection fraction- 35\%) and normal coronaries (RCA and LMCA at $+1.5 \mathrm{Z}$ score, $\mathrm{LAD}+1.7 \mathrm{Z}$ score). Chest $X$-ray showed cardiomegaly (Fig. 1) and cardiac enzymes [HS Troponin I- $29 \mathrm{ng} / \mathrm{L}(0-19)$, proBNP- $8000 \mathrm{pg} / \mathrm{ml}$ ] were elevated, suggesting myocarditis. Inotropic support with adrenaline was started and respiratory support with high flow nasal cannula (HFNC) $2 \mathrm{~L} / \mathrm{kg}$ flow was initiated. Intravenous antibiotic-ceftriaxone was also started. Overall constellation of clinical features (sterile pyuria, bulbar conjunctivitis, extremity edema, elevated ESR and CRP, hypoalbuminemia, myocarditis) suggested atypical KD. IV immunoglobulins $2 \mathrm{~g} / \mathrm{kg}$ was given over $18 \mathrm{~h}$. In view of symptomatic myocarditis in $\mathrm{KD}$, methyl prednisolone pulse $(30 \mathrm{mg} / \mathrm{kg} / \mathrm{d}$ for $3 \mathrm{~d}$ ) was also given. Diuretics for preload reduction, enalapril for afterload reduction and remodelling were also started. Daily monitoring with functional echocardiography showed improvement in left ventricular function. Perfusion improved gradually, inotropes and HFNC were tapered and stopped on day 3 of hospital stay. Serum creatinine normalised with the resolution of shock. Child remained afebrile from $24 \mathrm{~h}$ after IVIg transfusion. Repeat CRP (13 mg/L) and Ferritin $(75 \mathrm{ng} / \mathrm{ml})$ on day 3 showed decreasing trend. Blood culture was sterile and antibiotics were stopped. 2D Echocardiogram on day 5 of hospital stay showed improved left ventricular function (Ejection fraction$60 \%$ ) with normal coronaries. Real time PCR for SARSCoV-2 was done for him twice during the hospital stay and it was negative. Multiplex PCR for other respiratory viruses (BioMerieux, USA) done to find any other viral etiology was also negative. Child was discharged on day 6 of hospital stay on anti-thrombotic dose of aspirin, maintenance dose of oral steroids and low dose enalapril.
He remained well and there was no periungual desquamation noted during his review visit one-week later.

\section{Discussion}

There is a growing global concern that a SARS-CoV-2 related inflammatory syndrome is emerging in children. Clusters of children from UK with this inflammatory syndrome had acute febrile illness with evidence of single or multi-organ dysfunction. Laboratory features were neutrophilia, elevated CRP and clinical features included abdominal pain, gastrointestinal symptoms, myocarditis and shock [4]. The present case also had almost similar laboratory parameters and clinical profile.

Multiple infectious triggers like adenovirus and coronavirus (New Haven coronavirus) have been inconclusively associated with $\mathrm{KD}$ in the past $[5,6]$. In the cluster of cases from UK, common microbial causes were excluded and SARSCoV-2 PCR testing was positive in few cases, while serological tests (possible preceding SARS-CoV-2 infection) were positive in some cases. In the present case, RT PCR was negative on two occasions. The patient hailed from a COVID hotspot area and his father had returned from an endemic foreign country two months back. Hence, past asymptomatic infection in the child was a possibility. Antibody tests could not be done due to the unavailability.

A diagnosis of "atypical" $\mathrm{KD}$ is made in patients presenting with atypical or unusual clinical features. Though some evidence of myocarditis has been seen in more than $50 \%$ patients in the acute phase of $\mathrm{KD}$, symptomatic myocarditis with cardiogenic shock is rare [7]. Available reports of such cases suggested IVIG resistance and good response to pulse steroid therapy [8]. As the index patient's hemodynamic condition was deteriorating, the authors used steroids from day 1 itself. There was considerable improvement in left ventricular systolic function, and the authors could reduce inotropic support and avoid invasive ventilation.
Fig. 1 Chest X-rays of child on day 1 and day 5. Note the cardiomegaly with left ventricular dilatation on day 1 , which improved by day 5

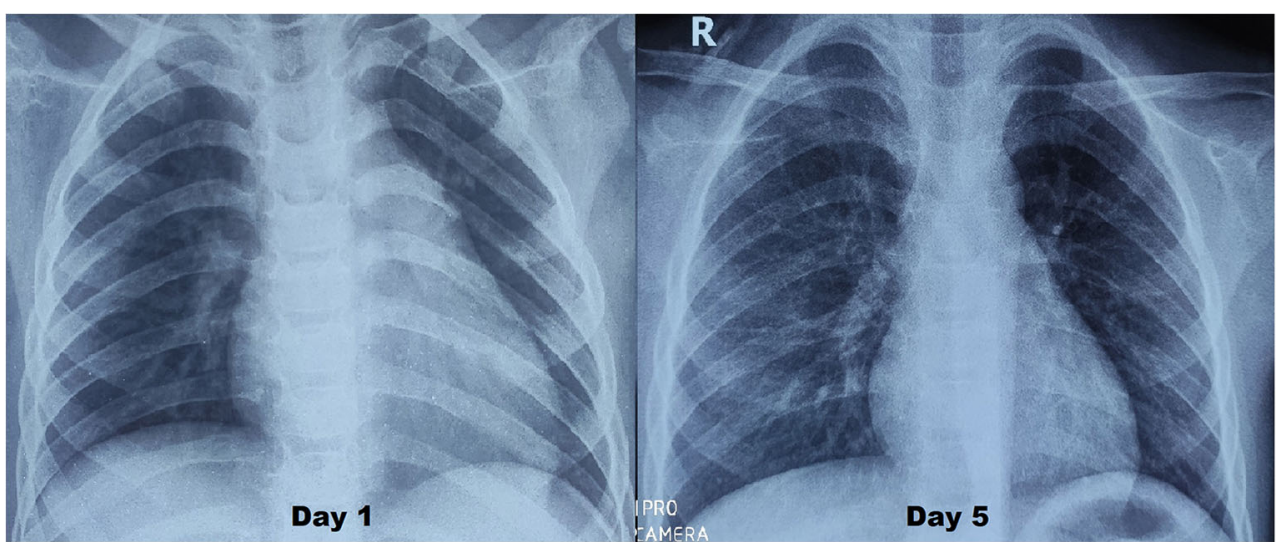


There could be different explanations for the possible association of COVID infection and KD. In PCR negative- antibody positive cases, it's quite possible that corona virus infection in recent past can trigger an immune response simulating $\mathrm{KD}$. COVID-19 is also known to cause excessive inflammation and cytokine storm like KD, during active infective phase. Similar alert regarding a mysterious inflammatory syndrome in children is now reported from USA also $[9,10]$. To the best of authors' knowledge, this is the first report from India about multi-system inflammatory syndrome simulating atypical KD during this pandemic. As of now, the association between COVID infection and KD is not conclusively proven. However, as similar reports have come from various countries with temporal relation to COVID infection in the community, general pediatricians need to be aware of such atypical presentations and early referral to tertiary care needs to be undertaken.

Acknowledgements The authors acknowledge Dr. Yassar Andru, Dr. Sayid Sabik, all Post graduate students in Dept of Pediatrics and nursing staff in Pediatric ICU for their involvement in patient care.

Authors' Contributions AR: Patient management, literature review and drafted the primary manuscript; AV: Patient management and revision of the manuscript; STJ: Revision of the manuscript; RAK: Patient care and revision of the manuscript; AL: Patient management. STJ will act as Guarantor of this paper.

\section{Compliance with Ethical Standards}

\section{Conflict of Interest None.}

\section{References}

1. Kawasaki T. General review and problems in Kawasaki disease. Jpn Heart J. 1995;36:1-12.

2. Burgner D, Davila S, Breunis WB, et al. A genome-wide association study identifies novel and functionally related susceptibility loci for Kawasaki disease. PLoS Genet. 2009;5:e1000319.

3. Pediatric Intensive Care Society. PICS statement: Increased number of reported cases of novel presentation of multi-system inflammatory disease. April 27, 2020. Available at: https://picsociety.uk/ wpcontent/uploads/2020/04/PICS-statement-re-novel-KD-C19presentation-v2-27042020.pdf. Accessed 02 May 2020.

4. Guidance- COVID-19 paediatric multisystem inflammatory syndrome. Royal College of Paediatrics and Child Health. Available at: https://www.rcpch.ac.uk/resources/guidance-paediatricmultisystem-inflammatory-syndrome-temporally-associatedcovid-19. Accessed 02 May 2020.

5. Jaggi P, Kajon AE, Mejias A, Ramilo O, Leber A. Human adenovirus infection in Kawasaki disease: A confounding bystander? Clin Infect Dis. 2013;56:58-64.

6. Esper F, Shapiro ED, Weibel C, Ferguson D, Landry ML, Kahn JS. Association between a novel human coronavirus and Kawasaki disease. J Infect Dis. 2005;191:499-502.

7. Dionne A, Dahdah N. Myocarditis and Kawasaki disease. Int $\mathbf{J}$ Rheum Dis. 2018;21:45-9.

8. Aggarwal P, Suri D, Narula N, Manojkumar R, Singh S. Symptomatic myocarditis in Kawasaki disease. Indian J Pediatr. 2012;79:813-4.

9. Jones VG, Mills M, Suarez D, et al. COVID-19 and Kawasaki disease: Novel virus and novel case. Hosp Pediatr. 2020. https:// doi.org/10.1542/hpeds.2020-0123.

10. New York City 2020 Health Alert \#13: Pediatric multi-system inflammatory syndrome potentially associated with COVID. Available at: https://www1.nyc.gov/assets/doh/downloads/pdf/ han/alert/2020/covid-19-pediatric-multi-system-inflammatorysyndrome.pdf. Accessed 02 May 2020.

Publisher's Note Springer Nature remains neutral with regard to jurisdictional claims in published maps and institutional affiliations. 